\title{
Finite Element Analysis for Quantitative Evaluation of a Transfemoral Prosthesis Socket for Standing Posture
}

\author{
Le Van Tuan \\ Shibaura Institute of Technology \\ 307 Fukasaku, Minuma-ku, Saitama-shi, \\ Saitama 337-8570, Japan
}

\author{
Shinichiroh Yamamoto \\ Shibaura Institute of Technology \\ 307 Fukasaku, Minuma-ku, Saitama-shi, \\ Saitama 337-8570, Japan
}

\author{
Akihiko Hanafusa \\ Shibaura Institute of Technology \\ 307 Fukasaku, Minuma-ku, Saitama-shi, \\ Saitama 337-8570, Japan
}

\begin{abstract}
The socket of a prosthesis is an important part that serves as the interface between the residual limb and the prosthesis. The soft tissue around a residual limb that is not well suited to load bearing and where an improper load is distributed may cause pain and skin damage. Correct shaping of the socket for appropriate load distribution is a critical process in the design of lower limb prosthesis sockets. In this study, a nonlinear finite element model was created and analyzed to evaluate the pressure distribution between a residual limb and the prosthesis socket of a transfemoral amputee. Three-dimensional models of the residual limb and socket were created using magnetic resonance imaging data; the models were composed of 21 layers, each separated by $10 \mathrm{~mm}$. Two types of socket MCCT socket and UCLA socket are used in this study for quantitative evaluation. The interface pressure distribution in the residual limb was observed in the same condition when the experiment of loading $50 \%$ and $100 \%$ of body weight and the pressure at eight locations on the surface between socket and residual limb was measured. The value of pressure between experiment and simulation got a high coefficient of correlation $(>0.9)$. This analysis allows prosthetist and engineers to simulate the fit and comfort of transfemoral prostheses in order to evaluate the fit of socket shape.
\end{abstract}

\section{General Terms}

Rehabilitation science, Welfare engineering

\section{Keywords}

Transfemoral prosthesis ,finite element analysis, UCLA socket, MCCT socket

\section{INTRODUCTION}

A lower-limb prosthesis is an artificial limb that is designed to mimic the natural function, structure, and aesthetics of a limb that is replaced. Different types of lower-limb prosthesis exist based on the levels of extremity of the lower-limb amputations. A transfemoral or above-knee prosthesis is an artificial limb for a case in which the knee joint is removed and part of the femur or thigh bone remains intact. The socket is one of the most important parts of the transfemoral prosthesis because it acts as a connection between the residual limb and prosthesis. It protects the residual limb and also appropriately transfers forces during standing and ambulation motions. The skin and soft tissue of a residual limb are subject to stress and excessive distortion during gait positioning [1], and are significantly higher during transfemoral prosthesis since a residual limb is comprised of complex soft tissue and experiences a large change in volume with the use of sockets. Thus, the prosthesis is unstable, and this makes it difficult for the patient. In order to evaluate the quality of socket design and fit, the pressure distribution at the interface between the residual limb and prosthetic socket is considered as an extremely important factor. An abnormal force transferred from the socket to a residual limb can cause unstable gait, pressure ulcers, and deep tissue injuries.

Several studies focused on quantifying the stress distribution at the interface between a residual limb and a socket. Mak [11], SilverThorn [16], Zachariah [20], Zang[21], and Minh Zang [8] employed either experimental measurements or finite element analyses to determine the stress distribution. Most studies examined transtibial or below-knee prosthesis. In the studies, the stress acting on the residual limb was easily measured by using finite element analyses in which the change in the volume of the soft tissue is low. This is because the shape of the socket is the same as that of the residual limb to a significant extent, and only a simple displacement occurs during the gait. Damien Lacroix and Juan Fernaldo Ramirez Patino [7] modeled the actual donning procedure of a socket for five transfemoral amputees and used finite element analysis to observe the stress distribution on the surface of stumps and bones. In the study, real models of a residual limb and socket were developed by using CT scan data in which the soft-tissue mechanical properties followed a hyperplastic condition, and the velocity of the residual limb was sufficiently low to reduce the dynamic effect. The residual-limb model included soft tissues and bones. The shapes of 


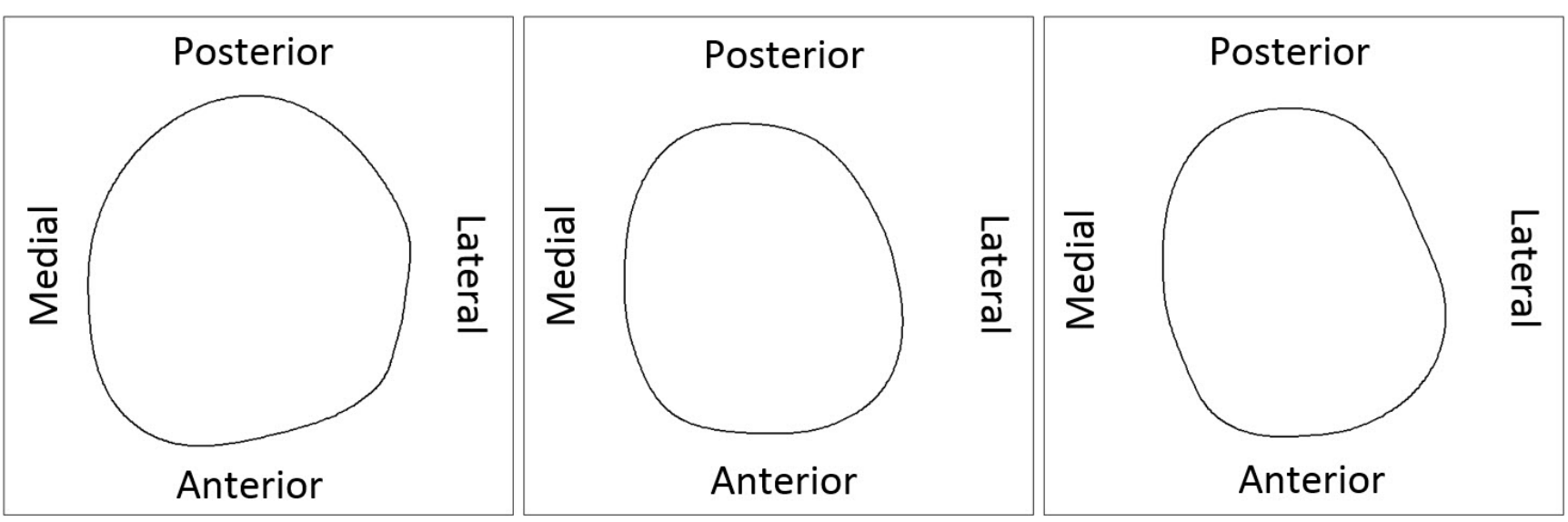

Fig. 1. Profile at the cross section from distal end of (a) residual limb, (b) UCLA socket, and (c) MCCT socket at $180 \mathrm{~mm}$

the socket and residual limb were the same to a considerable extent. Thus, the maximum contact pressure observed in the study corresponded to only $5.6 \mathrm{kPa}$, which was lower than that observed in other studies. Linlin Zhang [9] proposed a nonlinear finite element model to investigate the interface pressure between an above-knee residual limb and a prosthetic socket. The model of the residual limb included bones and soft tissues. The length of the model was significantly full until the point above the hip joint. The maximum normal stress corresponded to $80.57 \mathrm{kPa}$ at the distal end of the soft tissue. Extant studies obtained different results with respect to the position of maximum stress on the residual limb. This could be because it was assumed that the shape of the residual limb was the same as that of the rectified socket. S.Portnoy [14] characterized the mechanical conditions of a muscle flap of a trans-tibial patient for static load bearing, and this corresponded to a case study on the behavior of the residual limb. The residual-limb model included bone, muscle flap, and skin. The peak interface pressure between the residual limb and socket was calculated as $65 \mathrm{kPa}$ by using finite element analysis, and this was in good agreement with the pressure obtained in the experimental measurements. S.Portnoy [15] quantified the internal strains in a trans-tibial prosthetic during load bearing with respect to the change in the residual limb. Juan Fernando Ramrez and Jaime Andrs Vlez [13] identified a contact-boundary condition between the bone and soft tissues in a transfemoral amputee that affected the stress and strain states of the residual limb. The study provided more information on the bone?tissue interaction and aided in achieving more realistic and accurate finite element models to compute the stress and strain states of the residual limb with a prosthetic.

In the study, the authors proposed an overall approach to evaluate the transfemoral prosthesis socket in a more efficient manner by using a finite element analysis. First, the models of the residual limb and socket were developed, and this was followed by conducting meshing and simulation via finite element analysis. Finally, the results of the analysis were compared with those of the experiment. The residual-limb model included four parts, namely, bone, muscle, fat, and skin, which were different when compared to those used in previous studies. Specifically, UCLA socket [5] and Manual Compression Casting Technique (MCCT) sockets [12] were used in the present study. The real shapes of the socket and residual limb are significantly different, and thus, the computation was more complex and required considerable time for completion. Furthermore, experiments were conducted to determine the pressure acting on the interface between the socket and residual limb. Eight threedimensional force sensors were placed on the surface of the socket to measure the forces generated on the skin of the residual limb. The results of the simulation and experiment obtained for the two types of sockets were compared and discussed.

\section{METHOD}

The study involved a male subject (age 35 ) with a left-side transfemoral amputation. The height and mass of the subject corresponded to $169 \mathrm{~cm}$ and $63 \mathrm{~kg}$, respectively, in the absence of the prosthesis. The prosthesis incorporated the following two types of sockets: UCLA and MCCT type, a Nabco prosthesis knee, and an Ottobock foot.

The UCLA socket involves an ischial ramal containment (IRC) type socket created by using contour adducted trochanteric controlled alignment method (CAT-CAM) as introduced by John Sabolich and inspired by Ivan A and Long C.P. Based on the theory of the UCLA-type IRC socket, a new MCCT-type IRC socket was developed in Japan by Iida. Originally, the shape of the socket was designed to mimic the cross-sectional shape of an UCLA socket. However, the stability in the anterolateral direction was unsatisfactory, and thus the outline shape was narrowed by directly applying a force to the residual limb while casting. A MCCT-type IRC socket was designed in which the stability is improved in the anterolateral direction as well as in the sagittal direction. Figure 1 shows the profile at the cross section from the distal end of the residual limb, UCLA, and MCCT socket at $180 \mathrm{~mm}$. With respect to the MCCT socket, the outline was narrowed in the anterolateral direction while the length was extended.

\subsection{Geometric Modeling}

The shape of the socket and residual limb were considerably different, and thus it was necessary to separately model the geometries of the socket and residual limb. A magnetic resonance imaging (MRI) method was used to obtain the data of the residual limb by considering the limb with and without the prosthesis socket. The obtained data were divided into 21 layers with a separation of $10 \mathrm{~mm}$ perpendicular to the axis of the residual femur (Figure 2a and 2b). A segmentation process was performed by using Matlab software to distinguish the individual parts, which include bone, muscle, fat, and skin, from the data of the residual limb without the socket. This was followed by approximating the boundaries of the parts in each 

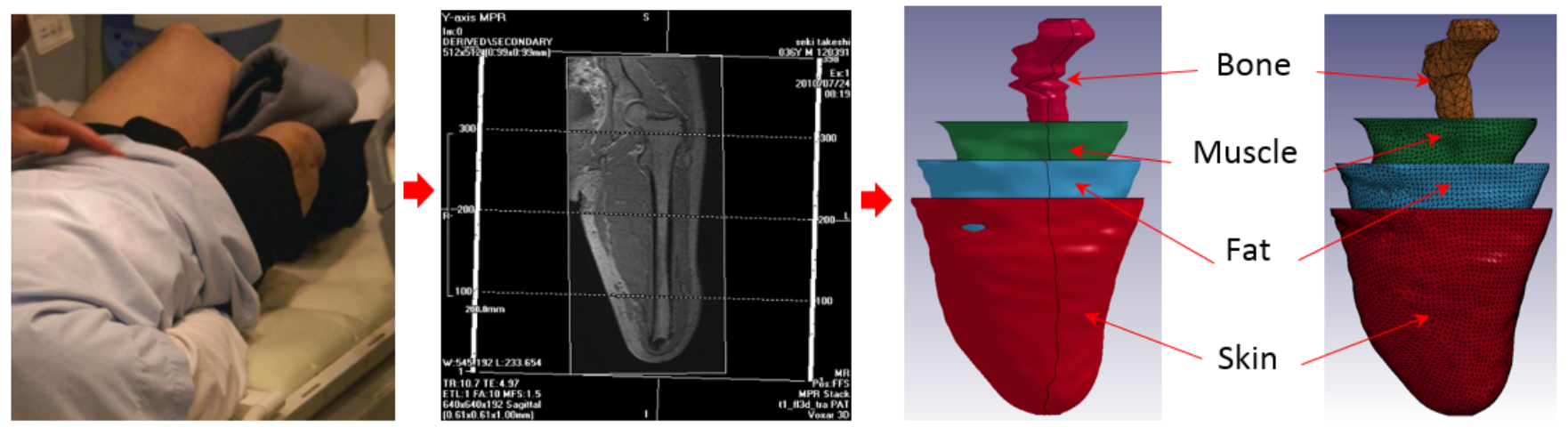

Fig. 2. Process for creating the model of residual limb. (a) Captured MRI; (b) Analyzing the MRI image; (c) 3D model; and (d) Finite element model.

MRI slice via a spline-curve method using the CAD software. The curve files were loaded on parallel planes again and incorporated in the 3D solid body by using a solid modeling software known as PTC Creo Parametric (Figure 2c). The boundary of the socket was unclear in the MRI image, and therefore the model of the socket was created by offsetting the skin shape of the residual limb in an outward direction up to the thickness of the socket.

\subsection{Finite Element Model}

\section{Element types}

The three-dimensional models were meshed using Hypermesh software (Altair Engineering). The socket was meshed as a shell element with a thickness of approximately $3 \mathrm{~mm}$. The bone and soft tissue including skin, fat, and muscle were meshed with the solid element, and its approximate size corresponded to $6 \mathrm{~mm}$. The type of solid element used corresponded to a tetrahedral (Figure 2d).

\section{Material model}

The mechanical properties of the bone and socket were assumed to be linearly elastic and obeying the Hooke?s law wherein the stress varies linearly with strain. The materials of the parts were modeled as isotropic with uniform elastic properties in all directions and were assumed as homogenous with consistent material properties. The femur bone was modeled with a Young's modulus of $17,700 \mathrm{MPa}$ and a Poisson's ratio of 0.3 [21]. The prosthesis socket was composed of acrylic plastic and was modeled with a Young's modulus of $1886 \mathrm{MPa}$ and a Poisson's ratio of 0.39 [18]. The soft tissue exhibited time-dependent behavior. Fung [19] proposed the quasi-linear viscoelasticity theory that is widely used in mechanics to describe soft tissue behavior. The main assumption of the theory corresponds to the convolution integral representation of the stress as shown in the following expression with respect to an uniaxial loading condition:

$$
\sigma(t)=\int_{0}^{t} G(t) \frac{\partial \sigma^{e}[\lambda(\tau)]}{\partial \tau} d \tau
$$

Where $\sigma^{e}$ denotes elastic response, $G(t)$ denotes relaxation function, and $\lambda(\tau)$ denotes stretch ratio time history. The soft tissue was considered a composite material that was comprised of collagen fibers embedded in a softer isotropic material referred to as the ground. Weiss [17] formulated the strain-energy function of the soft tissue material with terms $W_{1}, W_{2}$, and $W_{3}$ as expressed in equation (2) given below:

$$
W=W_{1}+W_{2}+W_{3}
$$

The first term $W_{1}$ aids in modeling the ground substance matrix as a Mooney-Rivlin material as follows:

$$
W_{1}=C_{1}\left(I_{1}-3\right)+C_{2}\left(I_{2}-3\right)
$$

Where $C_{1}$ and $C_{2}$ denote invariants of the right Cauchy deformation tensor.

The second term $W_{2}$ is defined to incorporate the behavior of the crimped collagen in tension, which works only in the fiber direction as defined in the model given below:

$$
W_{2}=F(\lambda)
$$

The role of the last term in the strain-energy function is to ensure that the material behaves in an incompressible manner to a significant extent, as given by the following.

$$
W_{3}=\frac{1}{2} K[\ln (J)]^{2}
$$

where $J=\operatorname{det} F$ denotes the third invariant of the deformation tensor, which changes based on the volume, and $K$ denotes the bulk modulus. The reduced relaxation function $G(t)$ represented by the Prony series is as follows:

$$
G(t)=\sum_{i=1}^{2} S_{i} \exp \left(\frac{-t}{T_{i}}\right)
$$

Here, $S_{i}$ and $T_{i}$ denote the spectral strength and characteristic time, respectively. In the study, $W_{1}$ and $W_{3}$ were used for skin, fat, and muscle [4, 3]. Table 1 lists the material properties of the skin, fat, and muscle.

\section{Contact definitions}

The first contact between the residual limb and socket was defined as a surface-to-surface contact. A coefficient of friction of 0.5 was assigned as an interaction property for the contact surfaces based on that used in another study [2]. The second contact definition applied corresponded to a tie contact between the bone and muscle, which is a simple way of bonding surfaces permanently and preventing slave nodes from separating or sliding relative to the master surface. This method of contact was obtained from another study [13]. The contact definition based on the hypothesis was applied to the connection between the skin and fat and fat and 

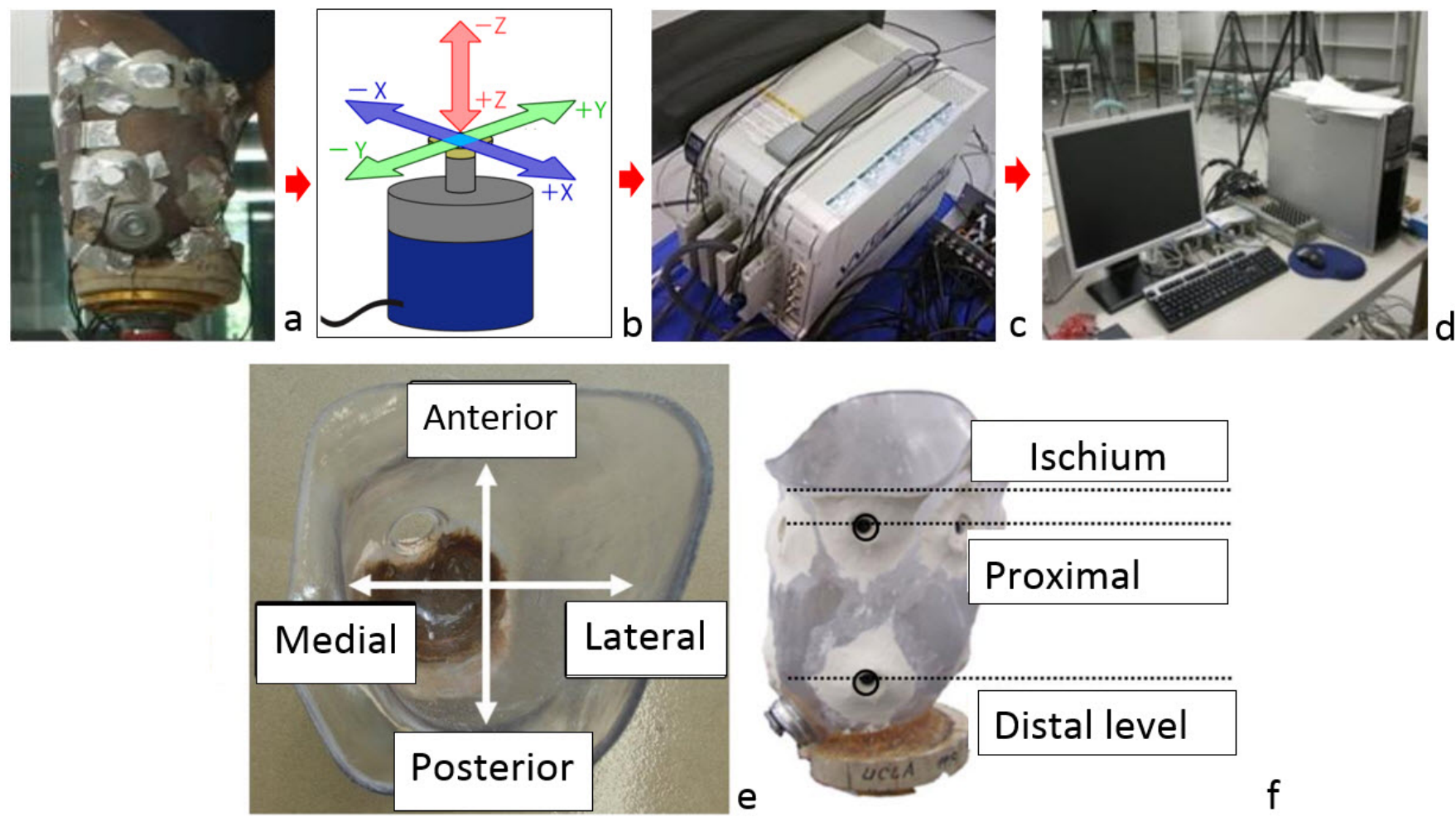

Fig. 3. A schematic of the experiment process (a: Sensors on the socket, b: Force directions, c: AD converter WE7000, and d: Computer) and defining the position of sensors (e: horizontal, f: vertical).

Table 1. Material properties of soft tissues

\begin{tabular}{ccccccccc}
\hline Name & $\begin{array}{c}\text { Density } \\
\left(\mathrm{kg} / \mathrm{m}^{3}\right)\end{array}$ & $\begin{array}{c}C_{1} \\
(\mathrm{kPa})\end{array}$ & $\begin{array}{c}C_{2} \\
(\mathrm{kPa})\end{array}$ & $S_{1}$ & $S_{2}$ & $\begin{array}{c}T_{1}(\mathrm{~ms}) \\
(\mathrm{ms})\end{array}$ & $\begin{array}{c}T_{2} \\
(\mathrm{~ms})\end{array}$ & $\begin{array}{c}K \\
(\mathrm{kPa})\end{array}$ \\
\hline Skin & 906 & 0.186 & 0.178 & 0.968 & 0.864 & 10.43 & 84.1 & 20000 \\
\hline Fat & 906 & 0.19 & 0.18 & 1 & 0.9 & 10 & 84 & 20000 \\
\hline Muscle & 1051 & 0.12 & 0.25 & 1.2 & 0.8 & 23 & 63 & 20000 \\
\hline
\end{tabular}

muscle in which the movement relation was neglected.

\section{Loads and boundary condition}

In the analysis, the residual limb was moved to the socket in the vertical direction. The socket was fixed in the vertical direction, and it only experienced displacement in the horizontal plane. The reaction force between the socket and residual limb was generated when the residual limb was put on the socket. The reaction force was equivalent to the load of body weight applied to the residual limb. The value of the force increased when the residual limb continued to move to the socket. When the value of the force was equivalent to half body weight and full body weight, interface pressure was observed between the residual limb and socket.

The analysis was conducted in the case of half and full body weights to model patients standing on two legs and a leg, respectively. At the end of the analysis, the shape of the residual limb was deformed and was fitted to the shape of the socket. In the first step, analysis was performed by considering the case including $50 \%$ body weight, and the stress and deformation were retained. A continuing analysis was performed with full body weight, and the stress and deformation were succeeded by the end of the analysis.

\subsection{Experimental Analysis}

Triaxial force sensors PD 3-32-05-015 NITTA Corporation [6 were used in the experiments. These force sensors help in resolving the force applied to the surface in the following three components: two shear components in the orthogonal direction (tangential to the skin surface) and a normal stress component (normal to the skin). Figure $3 \mathrm{e}$ and $3 \mathrm{f}$ show schematics of the installation area in which the sensors are installed. Eight sensors corresponding to eight areas of the socket were measured in the following two levels in the vertical direction: proximal level and distal-end level and in the following four directions in the horizontal direction: anterior, posterior, medial, and lateral directions. The eight area positions corresponded to AP (Anterior Proximal), PP (Posterior Proximal), MP (Medial Proximal), LP (Lateral Proximal), AD (Anterior Distal), PD (Posterior Distal), MD (Medial Distal), and LD (Lateral Distal). Figures 3a, 3b, 3c, and 3d show the schematic of the experiment and include the sensors placed on the socket of the patient, analogue-to-digital converter, data acquisition software, and computer, respectively. Figure $3 \mathrm{~b}$ shows the direction of force along the three axes of the sensor. The sampling frequency corresponded to $1000 \mathrm{~Hz}$. After measuring the forces, the pressure is calculated by 

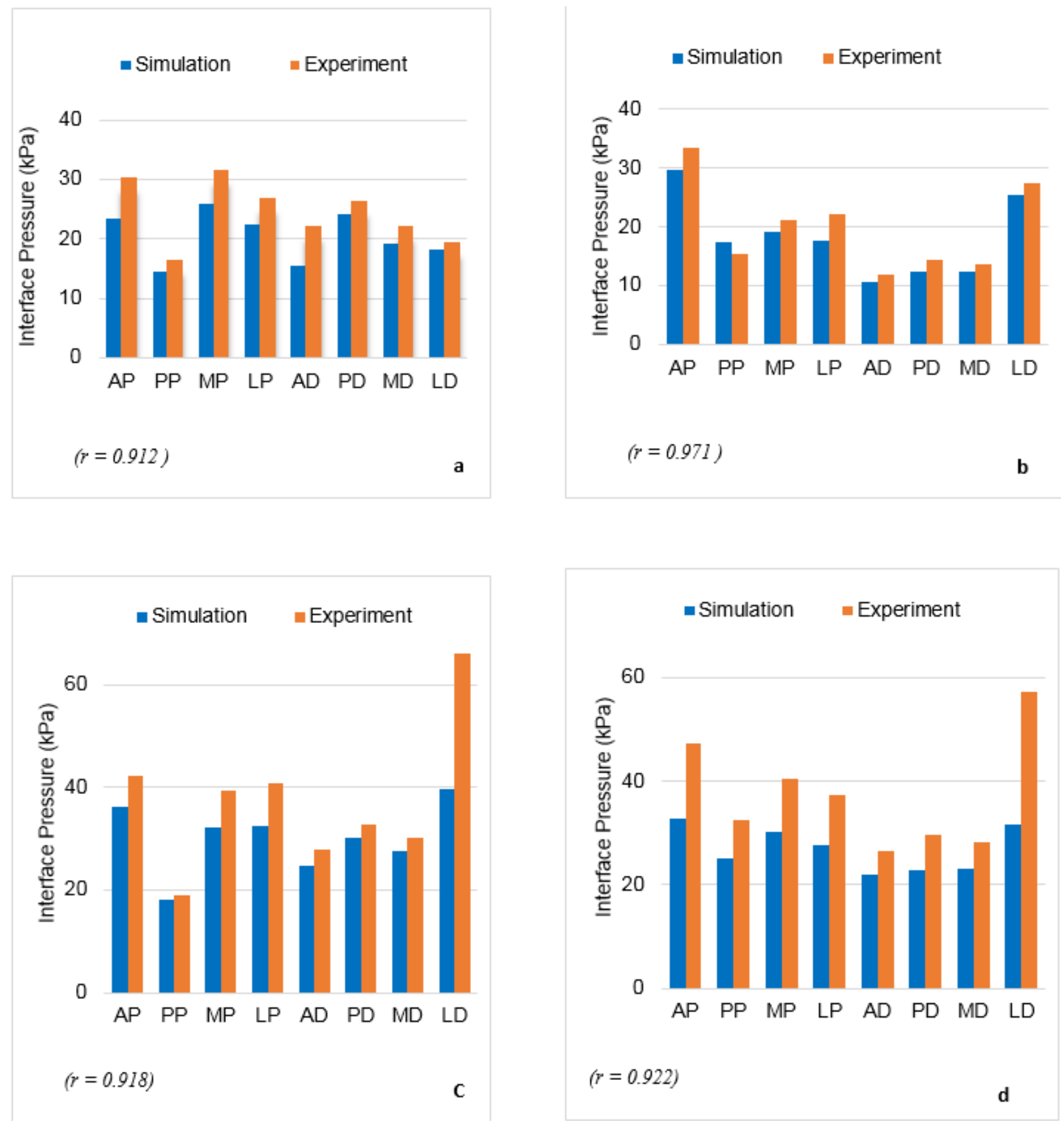

Fig. 4. Interface pressure at sensor locations in the experiment and simulation for sockets (a,c) MCCT and (b,d) UCLA with 50\% body weight (a,b) and $100 \%$ body weight $(\mathrm{c}, \mathrm{d})$.

using equation (7) as follows:

$$
[k P a]=\frac{V}{C} \frac{g}{4.5^{2} \pi 10^{-12}}
$$

where $g$ denotes the acceleration due to gravity, 4.5 denotes the radius of the sensor surface, $V$ denotes the voltage generated, and $C$ denotes the calibration coefficient.

\section{RESULTS}

Figure 4 shows the results of the pressure in the experiment and simulation for body weights of $50 \%$ and $100 \%$ corresponding to patients standing on two legs and one leg, respectively. The results also considered the two types of sockets. Generally, the pressures increased when $100 \%$ body weight was loaded when compared to 


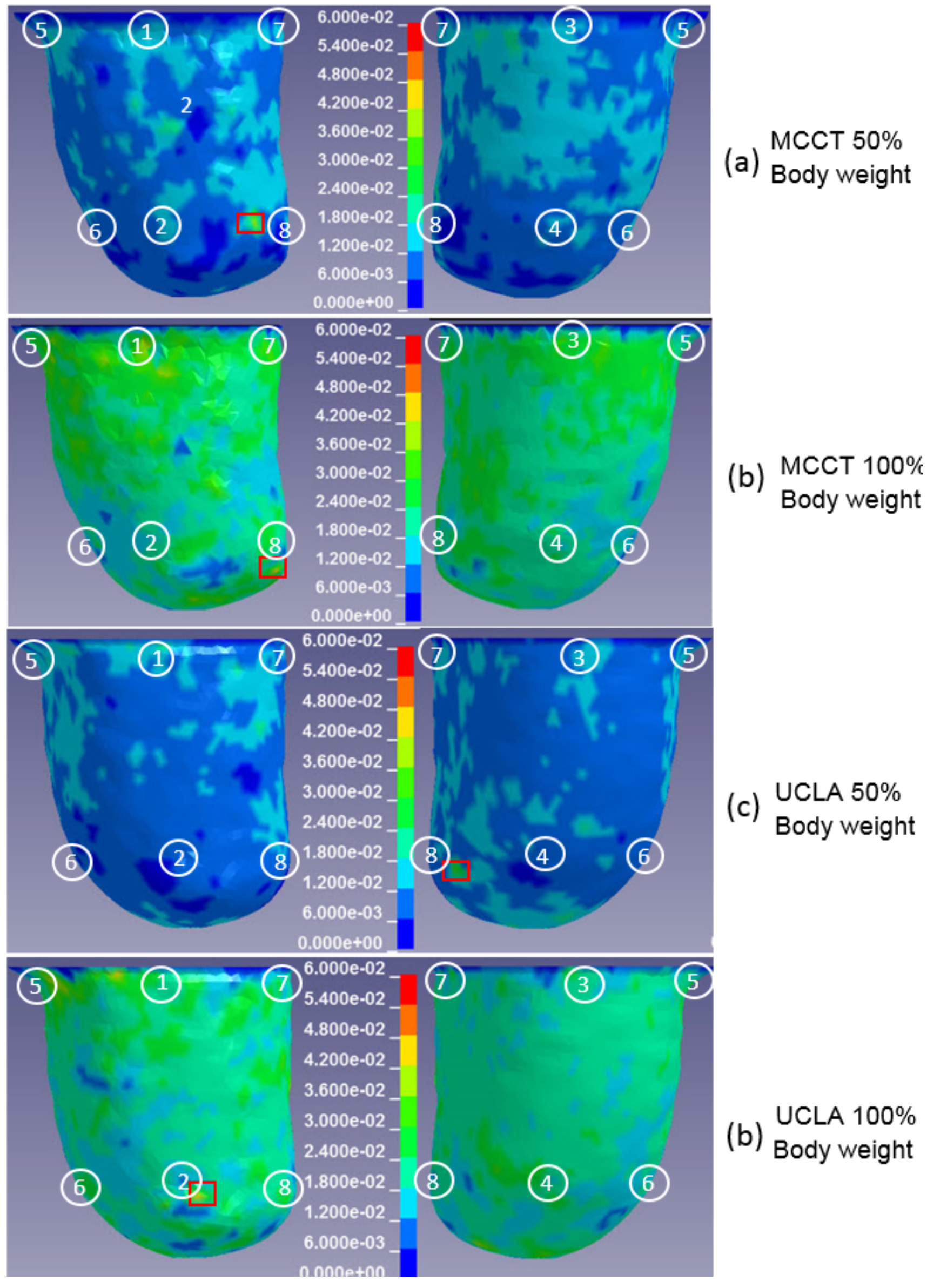

Fig. 5. Distribution of interface pressure (Unit: MPa). Anterior (left side) and posterior (right side) view. Red rectangles show positions where the maximum pressure is observed on residual limb. White circles 1,2, 3, 4, 5, 6, 7, and 8 show the position of sensors at AP, AD, PP, PD, MP, MD, LP, and LD, respectively. 
that when $50 \%$ body weight was loaded. The distribution of pressures for each sensor when MCCT and UCLA sockets were used was compared. However, when $50 \%$ body weight was loaded, pressures at MD, LP, AD, PD and MD were significantly higher in the case of the MCCT socket when compared to those of the UCLA socket. The fore-mentioned trends coincided with simulation and experiment results.

The pressures obtained in the experiment and simulation were different at $50 \%$ body weight when the MCCT socket was employed. In the AP, MP, LP, and AD areas, the pressure obtained in the experiment exceeded that in the simulation by $29.87 \%, 22.89 \%, 19.2 \%$, and $43.43 \%$, respectively. In case of the UCLA socket, the variation in the pressure is insignificant. In the AP and LP areas, the pressure obtained in the experiment exceeded that in the simulation by $12.21 \%$ and $17.14 \%$, respectively.

The correlation coefficients of pressure for the experiment and simulation in case of $50 \%$ body weight corresponded to 0.912 (Figure 4a) and 0.971 (Figure 4b) in the case of MCCT socket and UCLA socket, respectively.

In the case of $100 \%$ body weight, the difference in pressures between the experiment and simulation cases increased in the LD area. The pressure obtained in the experiment exceeded that in the simulation by $66.64 \%$ and $80.52 \%$ in the cases of the MCCT and UCLA sockets, respectively. In other area, the pressure obtained in the experiment exceeded that in the simulation. The correlation coefficients of pressure in the experiment and simulation correspond to 0.918 (Figure 4c) and 0.922 (Figure 4d) in case of the MCCT and UCLA sockets, respectively.

Figures 5 show the distributions of pressure on the surface of skin in the cases of MCCT and UCLA sockets for the body weights corresponding to $50 \%$ and $100 \%$. The distribution of pressure on the surface of the skin was clearly observed. The pressure and its corresponding location were easily determined. The maximum pressures on the surface of the residual limb in case of the MCCT and UCLA sockets for $50 \%$ body weight corresponded to $52.54 \mathrm{kPa}$ and $44.98 \mathrm{kPa}$, respectively. The maximum pressures on the surface of the residual limb in case of the MCCT and UCLA sockets for $100 \%$ body weight corresponded to $56.46 \mathrm{kPa}$ and $54.10 \mathrm{kPa}$, respectively. The positions of the maximum pressure area are indicated by red rectangular area in the figures.

\section{DISCUSSIONS}

The tendency exhibited by the pressures obtained in the results in cases of MCCT and UCLA socket are almost the same for both half body weight and full body weight conditions. This indicated that the shape profile of the two types of sockets ensured that the behavior of the residual limb is almost identical.

The differences between the results of the simulation and experiment were observed in all locations at the sensors. However, the values exhibited high correlation. A significant difference was observed in pressures $(66.64 \%$ and $80.52 \%$ for MCCT and UCLA sockets, respectively) at the lateral distal (LD) area for the $100 \%$ body weight. Nevertheless, the difference was negligible with respect to the $50 \%$ body weight. The reason for these differences could cause the differences in the model of geometries between the experiment and simulation or some abnormality in reality with respect to soft tissue features. The mechanical properties of the soft tissue changed based on age, sex, and health, and it was not uniform across the entire residual limb. Hence, the mechanical properties required for simulation were potentially not suitable for specific patients and specific areas. Currently, material models for soft tissues are developed to adapt to these features. The accuracy of the sensor and the method in which the sensor is positioned on the socket may lead to differences in the results.

In other areas of the residual limb that were not measured by the sensor, the distribution of pressure was clearly observed by the simulation results. The results showed that the distributions of pressure on the surface were almost identical in the cases of MCCT and UCLA sockets.

\section{CONCLUSIONS}

The main objective of the study involved enhancing existing methods by using finite element analysis to allow health-care providers and engineers to simulate the fit and comfort of transfemoral prosthetics. In the study, a model of a residual limb was initially developed with four parts, namely skin, fat, muscle, and bone. Thus, the model of soft tissue in the residual limb was more realistically represented, and this made it more precise with respect to the simulation processes. Second, the real shapes of two types of sockets were considered in the method. In most previous studies, the shape of the socket model was assumed as the same as that of the residual limb or an unrealistic socket model was created using software. The study used a real model of the two types of sockets, and the shape of the residual limb was significantly higher than that of the socket. This increased the complexity of the simulation because of the large deformation that occurred in the residual limb when it was put on the socket. The shape of the residual limb changed with respect to the shape of the socket and under a load of body weight, and thus pressure was generated on the surface of skin. The maximum value, position, and direction of pressure were easily observed. Furthermore, an experiment was conducted to confirm the effectiveness of the method. Two levels and four positions in the socket were selected to install the sensors. These positions represented the distribution of pressure on the surface of the skin.

The results showed that the correlation between the experiment and simulation exceeded 0.9 , thereby indicating a good agreement. It was assumed that the results of the simulation were equal to that of the experiment if the material properties of the soft tissue were more suitable for patients in the study. In the study, the pressure obtained in the experiment was consistently higher than that in the simulation and particularly at the LD (lateral distal) location. The observed pressure levels were significantly lower than the pain threshold limit that corresponded to $690 \mathrm{kPa}$ [10].

A comparison of the results of the two types of sockets showed that the distribution of pressure on the surface of the residual limb in the case of the MCCT socket was similar to that of the UCLA socket to a significant extent. The method for creating the MCCT socket shape involves less time for repairing and editing the shape of positive models of socket and therefore is convenient for a prosthetist with limited experience.

The results of the current study along with that of previous research studies indicate that the finite element analysis is a suitable method for evaluating prostheses. In the next study, it is intended to consider a specific subject in the material model to develop more advanced FE models of the transfemoral prosthetic-limb interface. Additionally, the accuracy of designing the 3D model of the residual limb should be improved.

\section{CONFLICT OF INTEREST}

The authors declare that there is no conflict of interest. 


\section{ACKNOWLEDGEMENTS}

The authors thank the members of the Prosthetic and Orthotic Group for their cooperation while performing the experiments.

\section{REFERENCES}

[1] S. Barbara and S. C. Dudley. Generic geometric finite element analysis of the transtibial residual limb and the prosthetic socket. Journal of rehabilitation research and development, 32:171-186, 1997.

[2] A. B. David C. L. Winson, Z. Ming and C. Bill. Finiteelement analysis to determine effect of monolimb flexibility on structural strength and interaction. J Rehabil Res Dev, 41(6A):775-86, 2004.

[3] Jeff Crandall Bing Deng J. T. Wang Costin D. Untaroiu, Kurosh Darvish. Development and validation of a finite element model of the lower limb. ASME 2004 International Mechanical Engineering Congress and Exposition Transportation: Transportation and Environment, Anaheim, California, USA, November 13 19, 2004.

[4] Jeff Crandall Bing Deng J. T. Wang Costin D. Untaroiu, Kurosh Darvish. Characterization of the lower limb soft tissues in pedestrian finite element models. Paper Number 050250, February 2005.

[5] C. Michael Schuch C.P.O. Report from international workshop on above-knee fitting and alignment techniques. Clinical Prosthetics and Orthotics, 12(2):81-98, 1988.

[6] NETTA Corporation RETS Division Sensor Group. Capacitive three axis force sensor. PFS series. PD 3-32 User's manual. NETTA Corporation, 2015.

[7] Damien Lacroix and Juan Fernaldo Ramirez Patino. Finite element analysis of donning procedure of a prosthetic transfemoral socket. Annals of Bioengineering, 39-12:2972-2983, December 2011.

[8] Ming Zhang;Winson C. C. Lee. Quantifying the regional load-bearing ability of trans-tibial stumps. Prosthet Orthot Int, 30-1:25-34, April 2006.

[9] Ling Shen Feng Zheng Linlin Zhang, Ming Zhu. Finite element analysis of the contact interface between trans-femoral stump and prosthetic socket. Conf Proc IEEE Eng Med Biol Soc, 2013:1270-3, 2013.

[10] A. Mak M. Zhang and W. C. Lee. Regional differences in pain threshold and tolerance of the residual limb: including the effects of age and interface material. Archives of Physical Medicine Rehabilitation, 86:641-649, 2005.

[11] Boone D A Mak A FT, Zhang M. State-of-the-art research in lower-limb prosthetic biomechanics socket interface. J Rehabil Res Dev, 38:161174, 2001.

[12] Japan Institute of Prosthetics and Orthotics Association. Manual Compression Casting Technique IRC Socket. Japan Institute of Prosthetics and Orthotics Association, 2015.

[13] Juan Fernando Ramrez and Jaime Andrs Vlez. Incidence of the boundary condition between bone and soft tissue in a finite element model of a transfemoral amputee. Prosthet Orthot Int, 36(4):405-14, December 2012.

[14] N. Shabshin et al. S. Portnoy, Z. Yizhar. Internal mechanical conditions in the soft tissues of a residual limb of a trans-tibial amputee. Journal of Biomechanics, 41(9):1897-1909, 2008.
[15] N. Shabshinc A. Kristalb Z. Yizhard A. Gefena S. Portnoy, I. Siev-Nerb. Patient-specific analyses of deep tissue loads post transtibial amputation in residual limbs of multiple prosthetic users. Journal of Biomechanics, 42(16):26862693, December 2009.

[16] Childress D S Silver-Thorn M B, Steege J W. A review of prosthetic interface stress investigations. J Rehabil. Res Dev, 33:253266, 1996.

[17] J.A. Weiss. A constitutive model and finite element representation for transversely isotropic soft tissues - Ph.D. Dissertation. University of Utah, 1994.

[18] C. C. Winson and M. Z. Lee. Design of monolimb using finite element modelling and statistics-based taguchi method. Clinical Biomechanics, 20:759-766, 2005.

[19] Fung YC. Elasticity of soft tissue in simple elongation. Am J Physiol, 213:1532-1544.

[20] Sanders J E Zachariah S G. Interface mechanics in lower-limb external prosthetics: A review of finite element models. IEEE Trans Rehabil Eng, 4:288302, 1996.

[21] Roberts V C Zhang M, Makaft. Finite element modeling of a residual lower-limb in a prosthetic socket: A survey of the development in the first decade. Med Eng Phys, 20:360273, 1998. 